\title{
Correction to: Left Ventricular Torsion Obtained Using Equilibrated Warping in Patients with Repaired Tetralogy of Fallot
}

\author{
Daniel Alexander Castellanos ${ }^{1}[1] \cdot$ Kateřina Škardová2 $\cdot$ Abhijit Bhattaru $^{3,4}(\mathbb{1}) \cdot$ Ezgi Berberoglu $^{5,6,7} \cdot$ Gerald Greil $^{4,8}$. \\ Animesh Tandon ${ }^{4,8}$ (1) . Jeanne Dillenbeck ${ }^{8} \cdot$ Barbara Burkhardt $^{9} \cdot$ Tarique Hussain $^{4,8}$ (D) $\cdot$ Martin Genet ${ }^{6,7}$ (1) \\ Radomir Chabiniok ${ }^{2,4,6,7,10}$ (1)
}

Published online: 9 November 2021

(c) Springer Science+Business Media, LLC, part of Springer Nature 2021

\section{Correction to: Pediatric Cardiology (2021) 42:1275-1283 https://doi.org/10.1007/s00246-021-02608-y}

In this article the affiliation details for 6 and 7 were incorrectly given.

The correct affiliation is given below.

(6) "Solid Mechanics Laboratory (LMS), Eìcole Polytechnique / CNRS / Polytechnic Institute of Paris, France" has been changed to "LMS, École Polytechnique, CNRS, Institut Polytechnique de Paris, Palaiseau, France"

The original article can be found online at https://doi.org/10.1007/ s00246-021-02608-y.

Daniel Alexander Castellanos

Daniel.castellanos@cardio.chboston.org

1 Department of Cardiology, Boston Children's Hospital, 300 Longwood Avenue, Boston, MA BCH 321502115, USA

2 Department of Mathematics, Faculty of Nuclear Sciences and Physical Engineering, Czech Technical University in Prague, Prague, Czech Republic

3 The College of New Jersey, Ewing, NJ, USA

4 Division of Pediatric Cardiology, Department of Pediatrics, University of Texas Southwestern Medical Center, Dallas, TX, USA

5 Institute for Biomedical Engineering, Swiss Federal Institute of Technology, Zurich, Switzerland
(7) "Inria, Le Chesnay-Rocquencourt, France" has been changed to "Inria, Palaiseau, France"

Publisher's Note Springer Nature remains neutral with regard to jurisdictional claims in published maps and institutional affiliations.
6 LMS, École Polytechnique, CNRS, Institut Polytechnique de Paris, Palaiseau, France

7 Inria, Palaiseau, France

8 Division of Pediatric Radiology, Department of Radiology, University of Texas Southwestern Medical Center, Dallas, TX, USA

9 Pediatric Heart Center, University Children's Hospital Zürich, Zurich, Switzerland

10 School of Biomedical Engineering \& Imaging Sciences (BMEIS), St Thomas' Hospital, King's College London, London, UK 